\title{
INDEX
}

aberrations

segregation, 243, 244, 253

X-chromosome, 200-201, 203-209, 2 I I

abscission layer, 428

acentric, $385,3^{86}$ sqq. 394-40I

Achillea, $3^{82}$

acquired character, 152

adaptation, $37,40,69,152,287$

adenine, $243,251,252-253,256,433,434$

agamospermy, 432

Aleurodiscus, 295

allelism, I, 3, 4, 5, 9, 10, 11 , 91

allelomorphs, 310 sqq., 333,357 sqq., 377 sqq., 433,434

Allen, 69

Allitu, 122, 392

alpha-propionic acid, 421

Altenburg, E., I 53-185

Altenburg, L. S., 153-185

amphibians, 120

analytical methods, 290

Aneilema, 141

angora in rabbit, 107 sqq.

anthesis, 422 sqq.

anthropology, 282-285

antibodies, 283,284

antigen, 283-284, 291, 354

apomixis, 431

apple-pear hybrids: $420-429$

Arabis, 306

ascospore formation, $243,244,246,248$ sqq.

ascus deviants, 297

Aspergillus, 177, 420-429, 434

Auerbach, C., 294

auto-segregation, $43^{\mathrm{r}}$

auxin, 427

backcrossing, I6, 1 7, 18, 107-1 10, 271, 435

bacteria, 35 sqq., 39-41, 43-44, 52-58

Bacterium prodigiosum, 177

Barber, 431

Barbosa, D., 227

barley, 351,428

basidium, 295

Bateman, A. J., 305-332

Bateson, W., 280

bee, 70

Bell, J., 147

Bergmann, 69

Bessel function, 188

biochemical character, 79

biometrical analysis, I5I

Biot, 285

biotin, 434

biotypes, 296, 432

blood groups in man, 68, $150,282-285$

polyploid cells, 12 I body size in Drosophila, 435

in mouse, $275^{-2} 78$

weight in mouse, 275,276

bollworms, see Diparopsis

Bonnier, G., 199-210

Brassica, 305

breeding system, 9 , I 1, 365

breoistylis in Einothera, 125 sqq.

Bridges, 280

Brock, R. D., 409-420, 42 I-429

Brunswick, 295

Buchholz, A., 289

bud pollination, 3

Butler, L., 275-278

Butlerov, 289

cabbage, 305

Cain, A. J., 286, 287

Callan, H. G., I52

Campanula, I4I

Campelia, 141

Canavalia, 140

cancer, $117,120-122,149,280$

in man, 118

in mouse, 120,121

in rat, 120

Capsella, 10, 305

Cardamine, 305

Carica, I4I

Catcheside, D. G., 125-137

centromere, $3^{89}$ sqq., 433

chaeta number, 13 sqq.

chemical phenotype, 377

protection, 207

Chi squared, 50, I1 0,1 32, 208, 21 3, 216-219 $249,25^{\circ}$

chiasma formation, 133-1 34,141

frequency, $152,294,410$

chimera, 301,421

Chlamydomonas, 295

chromosome aberrations, 200, 207, 21 7, 221, 414

breakability, 2 I I-223

breaks, 199, 207, 208, 21 1-222, 292, 385-407

constancy, I 1 7-124

fifth, in rabbit, 1 $07-1$ I 6

maps, 243, 433

pairings, $127-129,13^{r-1} 3^{6}$

systematics, 431

chromosomes, I 17, 279, 293

somatic, 117

spermatozoan, 199, 206, 207, 209

sex, 117,151

Cienfugosia, 228, 229

Clifford, H. T., 259-269, 434 
clines, $69,71,287$

clinical genetics, 149

medicine, 282-285

clover, see Trifolium

coat colour dilution, $275^{-278}$

Cocks, B., 13-34

coefficient of variation, 26,372

Colias, 69

Commelinacee, 145

comparative genetics, 327

compatibility, I sqq., $35^{8}$

complementary control, 252 genotype, $3^{16}$

Composite, 1, 305

Comstock, R. E., 365-376

Coprinus, 295

copulation of diploid cells, 255

correlated effects, 370

correlation of characters, $3^{2}$

corolla tube, 70

Cosmos, I-I I, 327

Costus sericeus, 139

cotton, 225

covariance analysis, 26, 27

Crepis, 1, 4, 9, 10, 11, 328, 389

Crosby, J. L., 152

cross-over, 89, 103, 104, 109, I I0-1 13, 189, 253, 273, 294, 410 sqq., 433

crossbreeding, 259

cross-incompatibility, 329

crossing technique, 246

Crowe, L. K., I-1 1, 357-363, 434

Crucifera, 305

cryptogam, 295

cultigen, 23I

culture methods, $14,16,36-37,47,80,82$

cytochrome bands, 81

cytodemes, 432

cytological markers, 401

cytoplasm, 434

cytoplasmic bodies, 147

effects, 435

environment, 333,353

heredity, 147, 296

proteins, $14^{8}$

Daday, H., 61-78, 377-384

Dalton, J., 287

Darlington, C. D., 279, 290

Darwin, 279, 285, 287, 289, 305, 431

De la Bathie, P., 232

Deol, M. S., 291

deviation, occasional, 248

systematic, $24^{8}$

dicentric, 404

Dickie, M. M., 271-273

differentiation, $4^{1} 5$

dilution in mouse, 275

Diparopsis, 228, 229, 239

disease resistance, I 50

dissociation, 53

Dobzhansky, T., 287 dominance, $1-11,17,333-336,435$

degrees, 368

relations, 311,327

variance, $365,369 s q q$.

Drosophila, I3-34, I 53-1 85, I 99-2 10, 21 I -223, $289-290,33^{8}, 351,352,3^{82}, 434,435$ embryonic development, 153

special stocks, I 59

Dubinin, 290

dwarf colony, 79-88

economics, 289

Edmondson, M., I $53-185$

education, 282

egg cytoplasm, 199, 219

hatchability, 292

organisation, 148

Ellenby, C., 434

Elliot, C. G., 294

embryo sac, 412-418, 422-424

embryos, fish, 120

mouse, 120

endometrium, I 1 7-1 I 8, 122

endomitosis, 255

Endymion non-scriptus, 294

energy levels 207-208

environment, I3, 22, 23,61, 64-68, IoI, $150,152,288,346$

enzymes, 252, 293, 377 sqq.

Ephrussi, B., 79, 82, 147

epistasis, 333, 374

Erophila, 431

Erysimum, 306

Escherichia coli, 177,178

ethoxy-caffeine, 385

Eucalyptus, 259 sqq.

euchromatin, $148,386,396$

evolution, $68-72,279,281,286-287$

Falconer, D. S., I51

fermenting ability, 246

Fernandes, 431

fertilisation, 424

fertility in Lilium, 409-420

field distribution, 259, 260, 262

Fincham, J. R. S., 293

fish embryos, 120

Fisher, Sir R. A., 89, 187-197, 276, 283, 284,337

fixation methods, 139, 422

flagella in Salmonella, 292

Flanders, 293

flocculent growth, 243

flower number, $338 s q q$.

Fonbrune's micromanipulator, 79,85

Ford, C. E., 386

fowl, 291

Freud, 280

Funaria, 295

Fungi, 149, 169, 433 
galactose, $244 s q q$.

Galeopsis, 350 sqq.

gametophyte, I, 434

gene action, 333

arrangement, $3^{82}, 3^{83}$

complexes, 159

conversion, 244

environment interaction, 333-356, 372

expression, 13,28

frequency, $61,64-78,377-384$

interactions, 324

order, 293

segregations, $25^{1-254}$

structure, 434,435

genes, 280,28 I

in enzyme production, 6r, 67, 293

genetic correlations, 373

ecology, 431

factors, 13,23

pathways, $352-354$

segregation, $43,57,125,130,135,13$ ?

structure, $379-382$

techniques, $158-160$

genic environment, 333

genotype, 29I

geographical variation, 68-72, 234

germplasm, 279

Gilmour, D. G., 291

Glass, D. U., 288

Gloger, 69

glucose, 79

glucoside, 61, 377, 378 sqq.

Glushchenko, I. E., 295

Godward, M. B. E., 293

gonadial mosaicism, 93, 99, I0 I

Gossypium, 225 sqq.

Gowen, 150

grading of material, 4I, 49

Green, 433

growth factors, 245-247, 253, 256, 293

Gudjonsson, 431

Guignard test, 377

Gulbekian, 221

gynandromorph, 205214

hair pigment, $3^{83}$

hairless, 1 5-33

Hanna, 295

haploid, 299, 301, 415

Hardy-Weinberg formula, 62, 64, 72, 377, 380

Helsinki, 306

Herskowitz, I. H., 289

Heslop-Harrison, J., 43I

heterochromatin, $148,220,386,396$

heterogeneity tests, 62

heterogenic tracts, 188, 190, 191, 194-196

heterokaryons, 293

heteromorphic plants, I, 10

heterosis, 333, 336, 374

heterothallism, 243

heterozygosity, I 52, 291, 335-336
Hollingsworth, M. J., 292

Homo sapiens, 97, $118-11$ 19, 282

cancer, 118

twins, 149, 288

uterus, I I 7, I 18, 122

homogeneity test, 23

homomorphic plants, I

homothallism, 243

homozygotes, 333-335

honey bee, see bee

Hooker, 285

hormone-induced hybrids, 420-429

Hottinguer H., 82, 243-258

house mouse, see mouse

Howard, A., 291

Hutchinson, J. B., 225-24I

Huyghens, 284

Hyacinthus, 411

hybrid, 248, 254, 264, 266, 268, 269, 420429,434

in Lilium, 409-420

swarms, 259-269

vigour, 365,435

hybridity, 152

coefficient, 410

hyperploid males, 200

Iberis amara, 305-332

identity tests, 98

illumination, 170

immuno-hematology, 282-285

inbred line, 276-278, 291, 292, 435

inbreeding, 151, 187-197, 269, 278, 338

incompatibility, $1,35,360,422,434$

in Angiosperms, 305-332

in Prunus, 357-363

indo-phenoloxidase, 81

infertility, 277

inflorescence, 339 sqq.

infrabar eye, $33^{8}$

infra-red irradiation, 207

interchange, 135

inter-family tests, 316,327

intersex, 205

intragenic changes, $211-214,220-221$

irradiation, 151, 197-210, 2 I1-223, 293

isogenic line, 435

isolates, 55,57

isotherms, $64-69$

James, W. O., 428

Janaki Ammal, E. K., 430

jerker, 29I

junctions, theory of, $187-197$

Kafer, E., 433

Kallmann, F. J., 288

karyotypes, 432

Kelton, D. E., 271-273

Kleczkowska, J., 36, 39, 43

Kniep, 295 
Landsteiner, 283

Laskowski, W., 79-88

Lavoisier, 285

leaf characters, 70,367

legal medicine, $282-285$

Leibnitz, 284

Lepeschinskaya, 289

Lepidium, 306

lethals, I 51 , 158 sqq., 211 , 212, 214 , 220, 221

Leupold, U., 82, 243-258

Levine, 283

Lewis, D., $333-35^{6} 357-363,435$

Lewis, E. B., 434

Life, Facts of, $279-282$

Liliacee, 294, 409-420, 421

linamarase, 61, 377

Lindegren, C., 299

linkage, 17, 32, 97, 101, 125, 148, 248, $264,278,374$

in the mouse, 271

in the rabbit, 107-115

Linnaeus, 431

Linum, 10

litter size, 291

Löve, 431

Luning, K. G., 199, 202, 21 1-223

Lycopersicon, 338 sqq., 342, 349, 354

Lyell, 285

Lysenko, 289

Lythrum, 10

McLaren, A., 291

McLeish, J., 385-407

maize, $3^{89}$

cob length, 266

pollen, 169, I 70, 177

maleic hydrazide, 385

Malthus, 288, 289

mammalian uterus, I 1 7-1 24

man, see Homo

Mann, T. J., 365-376

Manton, I., 431

markers, 21 5, 244-248, 255, 385, 401, 433

Marx, 28o

Mather, K., I51, 337

mating, $17,48,50,54$

groups, 2-7, II

response, 301

types, 193, 245

medicine, 149

megaspore, 422 sqq.

meiosis, 133, 152, 244, 294, 410, 422, 433

melanin, $3^{8} 3$

melibiose, 244

Melicocca, 140

Mendel, 280

Mendelian law, 295-304

menstruation, 118

mental disorder, 288

methionine, 243, 251-254

Meyer, H. U., I 53-185
Michie, D., 29r

Michurin, 289

microdissection, $82,84,85$

micronuclei, 385, 386 sqq., 394-401

micro-organisms, 147-149

Microtus agrestis, 118 , 119 , 122

Mielnicky, A., 118

mitosis, I 18-1 22, 293, 302, $3^{88}$ sqq., 412, 433

modifiers, 30,70

Morgan, T. H., 280, 295

mosaicism, 93, 205

Mourant, 283

mouse, 30, 89 sqq., 162-163, 218-220, 291

chromosomes, 195

embryos, 120

polyploid embryos, 120

selection, 151

Muller, H. J., 153-1 85, 280, 289, 290

multiple allelism, 97, 99, 10 , 284

genes, $243,248,251$

Mus musculus, see mouse

Musa, 14I

mustard gas, 294

mutagenic action, 79-85

efficiency, $165,169,173,175,18$ i

mutant clusters, $154^{-1} 56,162,164-168,172$, 178,180

cytoplasmic, 86

frequencies, $361-362$

genes, 13, 14, 18, 22, 28, 33, 125, 359, 435

processes, 199-210

respiration deficient, 79,87

runs, see clusters

spontaneous, $82,84,86$

types, $361-362$

mutation, 79-88, I5 I, I 53-185, 435

in Prunus, $357-363$

rates, $151,153-18_{5}$

revertible, 362

Nadi's reaction test, $8 \mathrm{r}, 84$

natural selection, $13,69,70,150,282,287$, 299

Nematode, 434

Neurospora, I 70, I 78, 293, 295

newt, 152

Newton, 284

Nicotiana, $1,3,6,7,9,10,307,338,342,349$, $352,354,365$ sqq.

nitrogen fixation, $35,36,5^{\circ}$

nodule isolates, 38

nucellus, 422

nuclear co-operation, 399

nucleo-cytoplasmic relations, 147-1 49

nucleolar organisers, 386,396

Nutman, P. S., $35-46$, $47-60$

nutrition, 435

Cinothera, 1, 9, 10, 125, 357, 359, 361, 432, 434

œstrus cycle, I I 7-I I9, 122 
Oparin, 289 ophthalmology, $15^{\circ}$ O'Sullivan, J., 118 oxygen tension, 207

Paramecium, 148, 354

Parnell, 229

Parthenium, 1, 4, 9, 10, 11, 328

parthenocarpic fruit, $421 \mathrm{sqq}$.

Partula, 69

Passiflora, 140

Pasteur, 285

Pavlov, 289

pear-apple hybrids, 420-429

Pearson, 229

Pedro, G., 228

perennials, 231

Peters, 283

Petunia, 351

phenotype, $61-62,7^{2}$

phenotypic equivalence, $89,100,104$ stability, 333-356

photorepair, 1 74-1 75, 1 76, 179

physiological balance, 353

picric acid test, 377

pig, 120

pigment formation, $245,252-253,256,434$

Planansky, 288

plant height, 367

parasite, 434

plasmagenes, 280

pleiotropy, $32,275-278$

Plumbaginacee, 305

Podospora, 148

Poisson series, 188, 292

pollen, 120, 125, $14^{\circ}$

behaviour, 1, 9

fertility, 409

maize, $169,170,177$

tube growth, $422 s q q$.

pollination, 2, 70, $306 \mathrm{sqq}$.

polydactyly, 147, 271

polygenes, $17,20,23,3^{\circ}, 3^{1}, 276$

polygenic character, 333

system, 13-34

polymeric genes, 243

polyploid cells, 121-123

embryos, 120

segregation, 244, 254-256

polyploidy, 244, 254, 296, 415,421

Pomoider, 421

Pontecorvo, G., 433, 434

populations, $64,72,287,288,329,377$

Priestley, 284, 285

Primula, 1, 10, $35^{1}$

Pritchard, R. M., 433

progeny test, 43, 47, 206

proline, 434

Prunus, 10, 357

Pryor, 329

pseudo-allelomorphs, 434

fertility, 5 psychiatry, 150, 288

Purser, A. F., 435

Pyrus, 42 I

quantitative inheritance, 151,365

Quintanilha, A., 295

Quixote, D., 283

rabbit, 107, 120

Race, 283, 434

radiation, 153

radish, 305

raffinose, 243, 397

Rattus norvegicus, 118

Raut, 79

Read, H., 280

reciprocal crossing, 4-7

recombinant genotypes, 254

recombination fraction, $107-112,115-116$

value, $132,134,136$

regression analysis, 20, 22, 27

coefficient, 67

resistance to tuberculosis, 288

reversion, 84

Rh factor, $97,282,285-286$

Rhizobium trifolii, 35, 36, $3^{8}$ strains, $36,37,38,39$

Rifaat, O. M., $107-116$

Roberts, C., 295-304

Robinson, H. F., 365-376

rogue tomato, 148

root nodules, $35,47,50,53,54$

tips, 139

Roux, 280

Rozi, 227, 230

Russian literature, 289

S-alleles, 3 1 0 sqq., 357 sqq, 435

complex, 434

genotype, 357

Saccharomyces, see yeast

Sachs, L., 11 7-124

Salmonella, 292

Sampford, M. R., 292

Sang, J. H., 435

Schizosaccharomyces, 243, 254

Schofield, 147

Scilla non-scripta, 294

Scitaminacea, 145

Scrophulariacea, 305

scute, 13-33

seed, $2,418-420$

segregation, 243-258, 269, 276, 291, 295

selected lines, 345

selection, 79, 174, 237, 291

experiment, $40,41,42$

factor, 87

germinal, 294

in mouse, 151

response, 151,287

self compatibility, $2,325,358,434$

sensitivity patterns, 294 
seriation, 20, 27 sqq.

serology, 282

Sesbania, I40

sex-linked lethals, I99, 206, 209

recessives, 17

sex-ratio, in man, 147

mouse, 291

Nematode, 434

Shull, G. H., I 25

sib mating, I $87,189,196,29$ I

" sifter" stock, I59, I60, I63, I 80

significance tests, I 5 I

Simmonds, N. W., I $39^{-1} 46$

Sisymbrium, 306

Skalinska, 43 I

Smith, J. M., 292

Smith, P., 271-273

Snell, G. D., $277^{-2} 73$

Solanacea, 305

Sorenson, $43^{1}$

Sorghum, 399

Sorsby, A., I 49

species concept, 238,287

specificity of strains, $5 \mathrm{I}$

spectroscopy, 86

Spemann, 280

spermatozoa, I5 I, 2 I I-223

Spirogyra, 293

spontaneous mutations, 357

sporophytic control, 3 I I

sporulation, 246, 254

stability factor, 334 sqq.

of strains, 54

Stadler, L. J., 434

statistical analysis, I 5I, I62, 206, 275

Stebbins, 43I

sterility, 125, 292

Stern, C., 43 I

Stocker, B. A. D., 292

Strelitzia, I40, I4I

Strickland, 433

Sturtevant, A. H., 280

subdiploid variation, I I 7, I I 8, I 20-I 2 I

sucrose fermentation, 243

superdominance, 336-338

symbiosis, 35, 36-39

synchronisation, 4 I 5

syndactyly, I 47

synergid, 412, 422

synthesising ability, 246

systematics, 286,431

\section{Taraxacum, 43}

Tephrosia, 140

tetrad, 249-250, 253, 254

analysis, 295-304, 433

tetraploid, I $39,256,4^{1} 5,421,435$

tetrazolium chloride, $79-88$

Thornton, H. G., 36, 39

Timiriazev, 289
Timofteff-Ressovksy, 290

Tischler, $43^{I}$

tobacco, see Nicotiana

tomato, see Lycopersicon

topoclines, 432

Tradescantia, $177,207,416$

transduction, 292, 293

transfusion reaction, 283,285

translocations, 152

Trichophyton, 169,170

Trifolium, I, I0, 35-46, 47-60, 6I-78, 377-384

triploidy, I 39-I 40, 244, 4I 5, 42 I

trisomics of $O$. blandina, $126-129$

in yeast, 244

Triturus, I 52

tropical plants, I $39^{-1} 46$

tryptophane, 249,251

tuberculosis resistance, 288

Turesson, $43 \mathrm{I}$

Turrill, W. B., $43^{1}$

twin studies, human, 149, 288

ultra-violet dose, I 53-1 85

Uvularia, 399

uracil, 252 sqq.

Ustilago, 295

uterus, mammalian, I I 7, I 8

variability, 22, 32, 7o, 98, 263, 333, 365

variance, 365,369 sqq.

variation, continuous, 13

in Bacteria, $35-36$

in host, $35-36$

viability, 220

Vicia, $3^{8} 5,389$ sqq.

viruses, 147,280

Wallace, 285

Wallace, M. E., 89-105

webbed toes, 147

Weismann, 279

Wettstein, 295

Wexler, 283

Whitbread, S., 289

Wiener, A. S., 282

Williams, R. D., 6I , 63, 67, 71

Winge, Ø., 295-304, 43 I

Woolf, B., I5I

X-radiation, I97-2 I O, 294, 357

Xanthosoma, I39

xerophyte, 23I

Yates, F., I 5 I

yeast, 79-88, I 48, 2 1 2, 243-258, 295

yellow, I $99 \mathrm{sqq}$.

$\mathrm{Yu},{ }^{2}{ }^{8}$

Zea mays, see maize

Zebrina, I4I

Zenker's fluid, 302. 